cases out of 56 cases, and the electroencephalographical examination was made in 31 cases of them. The recordings were interpreted as "WNL" in 7 cases, "OBL" in one case, and "abnormal" in 23 cases.

Postperative electroencephalogram showed no significant changes to preoperative recordings in the mojority of cases, even in the cases who were alleviated by surgery and had no clinical symptoms and deficits. Now, as the surgical results, no clinical symptoms and deficits were obtained in 20 out of 28 cases who were operated within 2 months after head injury. However, several deficits, such as motor disturbance, mental retardation or clinical seizures, were persisted in 9 cases out of 18 cases who were operated 2 months or more after head injury. It is, therefore, emphasized that early diagnosis is the most influencing factor to their prognosis of surgery and the electroencephalographic examination is one of the useful choice for this early diagnosis. However, electroencephalographical following was not correspond to the clinical feature after surgey.

\title{
G-3. Subdural Haematoma in Infant
}

\author{
Ikeo Mizawa, Yukinori Furuta, Shigeo WaKabayashi, \\ Masatoshi Ozawa, Takuji Takagi and Ryoji NagaI \\ 2nd Department of Surgery, Nagoya City, University of Medicine
}

We reviewed the clinical symptomatology of subdural haematoma in infant and pertient $\mathrm{x}$-ray findings and diagnostic methods, and reported the results in 11 patients operated on. There were 6 males and 5 females, who ranged in age from 3 days after birth to 11 months.

Contributing causes were cerebrocranial trauma (4), abnormal labour (5), and unknown (2). The cerebrocranial trauma was slight or moderate in all 4 cases. The abnormal labour was vacuum delivery, cesarean section owing to contracted pelvis, early rupture of the fetal membrane, cesarean section owing to weakness of labour pain. Of these 5 patients, four suffered from severe cerebrovascular insufficiency, which was asphyxia (3) and cyanosis of long duration (1).

The most initial symptoms were convulsion (8), weak cry and difficulty in feeding (1), unconsciousness (1), and vomiting (1). The most common clinical signs and symptoms included convulsion (8) [generalized-6, focal-1, focal and generalized1], vomiting (8) cloudiness of consciousness (8), weak cry (6), difficulty in feeding (4), bulging fontanelle (3), anaemia (3), hemiplegia (2), pathological reflexes (?). Of the 5 patients investigated neuroophthalmologic aspects, retinal haemorrhage was revealed in 3 , and papilloedema in 1 .

All haematomas were located supratentorially, but not infratentorially. Bilateral haematomas were found in 8 , and unilateral one in 3 . The conditions of thse haematomas were solid (1) and liquid (10). The capsule of the haematomas was definitely formed in 7 and not in 4 . 
Diagnosis was made by the findings of neurological signs and symptoms and by the other examinations. Roentgenography was done in all cases but none of them suffered from any type of the skull fracture. Angiography was performed in 10 and revealed avascular area over the surface of the cerebral cortex in the fronto-temproro-parietal region in all these patients. Some of them showed the additional extension of the avascular area at the base of the temporal lobe. Echo-encephalography was done in 10, and revealed deviation of the mid-line echo only in 2. MacEwen's sign was positive in all patients, and especially 9 of them revealed clearly to be in tympanic tone over the subdural haematoma. We stressed that MacEwen's sign was one of the most available methods to the diagnosis. Some cases were investigated by exploratory burr holes, air study, E. E. G., lumbar puncture, subdural tap, brain scanning, transillumination, and etc..

All patients were operated under intratracheal general anaesthesia. Some of exploratory burr holes were made in all cases bilaterally. In cases without capsule of the haematoma, its content was removed from the burr holes. But in patients with capsule of the haematoma, osteoplastic craniotomy was performed and both inner and outer membranes of the capsule were removed partially but as possible as extensively. All patients made definitely improved after operation.

\title{
G-4. A Consideration on the Therapy of Chronic Subdural Hematoma of Infancy and Ghildhood
}

\author{
Zenji Matsushima, Kunio Hashimoto and Yutaka Inaba \\ Department of Neurosurgery, Tokyo Medical and Dental University
}

\section{G-5. Traumatic Intracranial Hematoma in Children}

\author{
Masayuki Matsushima, Shozo Shimada, Masao Motomochi, \\ Toshio Ametani, Kazo Sato, Sachio Nabeshima, \\ Makoto Kako, Yoichi Suzuki, Hiroshi Oka, \\ Toyoshiro Yamamoto and Kyozo Ando \\ Depariment of Neurosurgery, Osaka Red Cross Hospital
}

Forty eight cases of traumatic intracranial hematomas in children were presented with special reference to their characteristics in pathological mechanism and clinical syndrome. They were admitted to Osaka Red Cross Hospital for last 8 years, and represented $11.2 \%$ of the total intracranial hematoma and $7.9 \%$ of all children hospitalized with head injury during this period. 